\title{
Multiple Fault Detection of Rolling Bearing through Ensemble Empirical Mode Decomposition of Vibration Signal
}

\author{
Sandip Kumar Singh
}

\begin{abstract}
Generally, two or more faults occur simultaneously in the bearings. These Compound Faults (CF) in bearing, are most difficult type of faults to detect, by any data-driven method including machine learning. Hence, it is a primary requirement to decompose the fault vibration signals logically, so that frequencies can be grouped in parts. Empirical Mode Decomposition (EMD) is one of the simplest techniques of decomposition of signals. In this paper we have used Ensemble Empirical Mode Decomposition (EEMD) technique for compound fault detection/identification. Ensembled Empirical Mode Decomposition is found useful, where a white noise helps to detect the bearing frequencies. The graphs show clearly the capability of EEMD to detect the multiple faults in rolling bearings.
\end{abstract}

Keywords: Compound Fault (CF), Empirical Mode Decomposition (EMD), Ensemble Empirical Mode Decomposition (EEMD), Intrinsic Mode Functions (IMF)

\section{INTRODUCTION}

Most of the rotating machine the failure is attributed to the failure of Rolling bearings. Rolling bearings fail due to the failure of generally four parts i.e., the outer race the inner race the roller, and the cage. When some failure occurs; a particular frequency is generated corresponding to that failure. If one type of failure persists for a longer time, it is responsible for another failure. This situation is termed as compounded fault situation. It is quite difficult task to detect compounded faults in bearings. Many techniques have been devised for detecting the single faults, but there are few which have been effective in case of compound faults. The most of the downtime of industries is caused by failure of bearings. It has great financial impact. The cost of bearings is not much high, but their failure causes great loss to the production efficiency.

Grasso M. et al [4], Hong et al. [5], and Wang et al. [6] utilized a data driven model for health assessment and life estimation of bearing using empirical mode decomposition (EMD) techniques. Ball fault frequency $f_{B D}$, Outer race fault frequency, $f_{O}$ and Inner race fault frequency $f_{I}$ are given as

$$
f_{B D}=\frac{P D}{2 * B D} f_{S}\left(1-\left(\frac{B D}{P D}\right)^{2} \cos ^{2}(C)\right)
$$

Revised Manuscript Received on December 30, 2019.

* Correspondence Author

Sandip Kumar Singh*, Department of Mechanical Engineering, V B S Purvanchal University Jaunpur, Uttar Pradesh (INDIA)-222001. Email: sandipkumarsingh25@gmail.com

(C) The Authors. Published by Blue Eyes Intelligence Engineering and Sciences Publication (BEIESP). This is an open access article under the CC BY-NC-ND license (http://creativecommons.org/licenses/by-nc-nd/4.0/)

$$
\begin{aligned}
& f_{O}=\frac{n}{2} f_{S}\left(1-\frac{B D}{P D} \cos (C)\right) \\
& f_{I}=\frac{n}{2} f_{S}\left(1+\frac{B D}{P D} \cos (C)\right)
\end{aligned}
$$

Where, $f_{S}$ is the speed of shaft rotor in $\mathrm{Hz}, \mathrm{BD}, \mathrm{PD}, \mathrm{n}$ and $\mathrm{C}$ are ball diameter, pitch diameter, number of balls in the bearing, and contact angle respectively. $\mathrm{C}$ is zero for ball bearings.

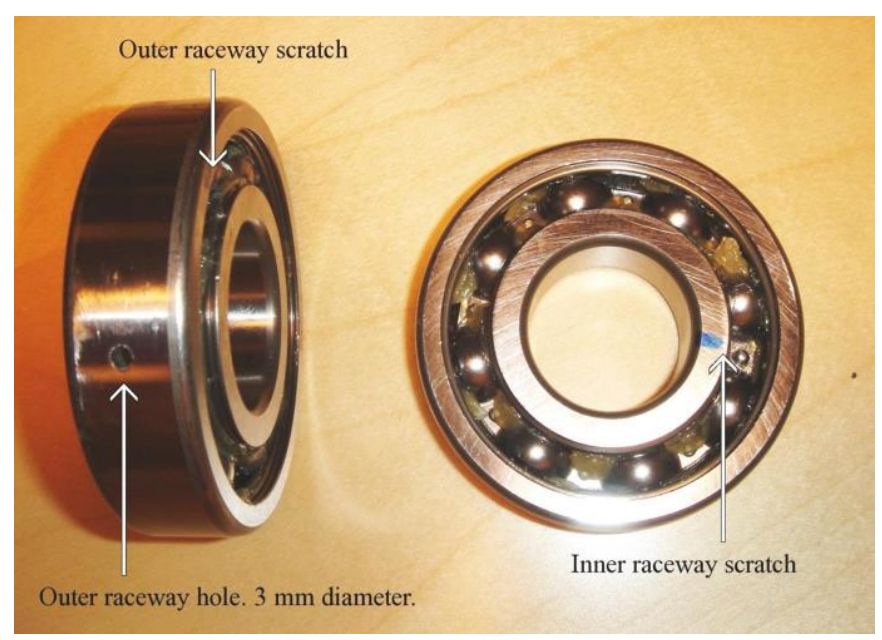

Figure1: Artificially created scratches on bearing parts

Fault detection of bearing can be done by vibration signal using signal processing.

The objective of bearing fault diagnosis is to examine if the vibration signal $x(t)$ consists of the bearing fault signal $f(t)$ faulty bearing signal can be expressed as $\boldsymbol{x}(\boldsymbol{t})=\boldsymbol{f}(\boldsymbol{t})+\boldsymbol{n}(\boldsymbol{t})$ normal bearing signal can be expressed as $x(t)=n(t)$,

where $n(t)$ is the noise, which is unknown. Faulty bearing signal is expressed as a modulated signal

$$
f(t)=m(t) c(t)
$$

$m(t)$ is the modulating signal. Its frequency component is the fault signature. The frequencies of bearing are made available by designer of the bearing.

$c(t)$ is the carrier signal.

The detection becomes to examine if the fault signature can be extracted from $\boldsymbol{x}(\boldsymbol{t})$

The important signal processing methods are as follows: 


\section{$\rightarrow$ Fast Fourier transform \\ $\rightarrow$ Wavelet transform \\ $\rightarrow$ High freq. resonance technique \\ $\rightarrow$ Empirical mode decomposition \\ $\rightarrow$ Envelop detection \\ $\rightarrow$ Adaptive noise cancellation \\ $\rightarrow$ Short-time Fourier transform \\ $\rightarrow$ Spectral kurtosis}

We propose Ensemble Empirical Mode Decomposition (EEMD) for multiple fault detection of bearing.

\section{THE PROPOSED METHOD}

A. Empirical Mode Decomposition (EMD): Empirical mode decomposition is it technique like Fourier transform or wavelet transform. It Breaks any signal into intrinsic mode functions (IMFs)which are nearly orthogonal bases. These IMFs are generally in finite numbers. The order of IMF follows from higher frequency to lower frequencies. Empirical mode decomposition is obtained by Hilbert-Huang Transformation (HHT).

EMD is used for decomposition of non-stationary \& non-linear signals. The non-stationary signals are those signals which change the mean and variance with time. Generally, the signals obtained from rolling bearing are of non- stationary nature. the intrinsic mode functions are different from simple harmonic function as they show variable amplitude and frequency unlike two simple harmonic functions. The process of obtaining IMFs from the given data is called shifting. The intrinsic mode functions are generated by following two simple conditions:

(a) At first, all local maxima and minima of data are identified.

(b) All local Maxima are connected to form an upper envelope, and all local minima are connected to form a lower envelope.

The upper and lower envelops are created in such a way that no data falls outside this envelope.

If the data is represented by $x(t)$ and mean of the data is $m_{1}$ then the difference of the two is called $h_{1}$, the component.

$$
h_{1}=x(t)-m_{1}
$$

Now $h_{1}$ takes place of $x(t)$. New mean becomes $m_{11}$ and the new component is $h_{11}$. Hence $h_{11}$ is written as:

$$
h_{11}=h_{1}-m_{11}
$$

Extending the same logic, we can write the kth component as follows:

$$
h_{1 k}=h_{1(k-1)}-m_{1 k}
$$

It becomes the first the intrinsic mode function and is written as: $c_{1}=h_{1 k}$

Original data $x(t)$ can be expressed as,

$$
x(t)=\sum_{i=1}^{n} c_{i}(t)+r_{n}(t)
$$

When we take the Hilbert transform of these IMFs, it provides the instantaneous frequencies in the vibration signals.

\section{B. Ensemble Empirical Mode Decomposition (EEMD):}

Ensemble Empirical Mode Decomposition is used for improving the performance of EMD. Mode mixing is a problem generally found in EMD is resolved by adding white Noise to the vibration signals. After addition of white noise, the signal is decomposed into intrinsic mode functions the process is repeated by bye adding a copy of White Lies noise and again doing the decomposition through EMD.

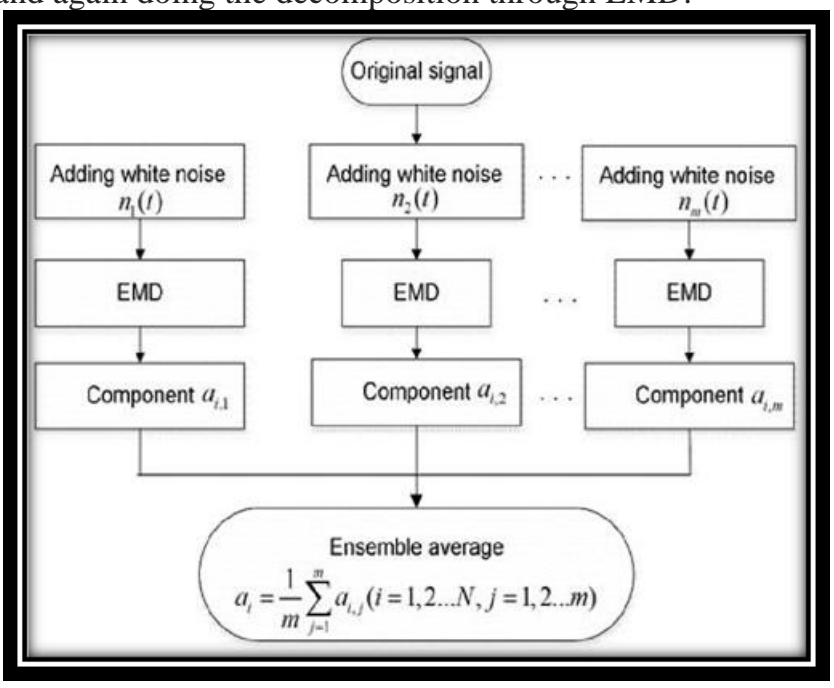

Figure2: EEMD Method

This is repeated number of times. At each time of decomposition fresh IMFs are generated. By taking the average of each IMF, the IMFs are finally created. The white Noise added to the raw data cancels one another in the process of averaging. Figure 1 shows the flowchart of EEMD.

\section{RESULT \& ANALYSIS}

The open access data set for validation of method has been used from PloSone [2]. The EEMD method has been tested by using for 500, 900 and $1300 \mathrm{rpm}$ datasets [2]. Figure 3 shows the original input signal used and different IMFs obtained. It is observed clearly that the fault signal is a superimposition of weak multiple signals and strong confusion noise. On decomposing the input signal using EEMD we obtained five IMF's. The FFT of the IMF- 04 in figure 4 shows the existence of outer race fault on $60 \mathrm{~Hz}$. 

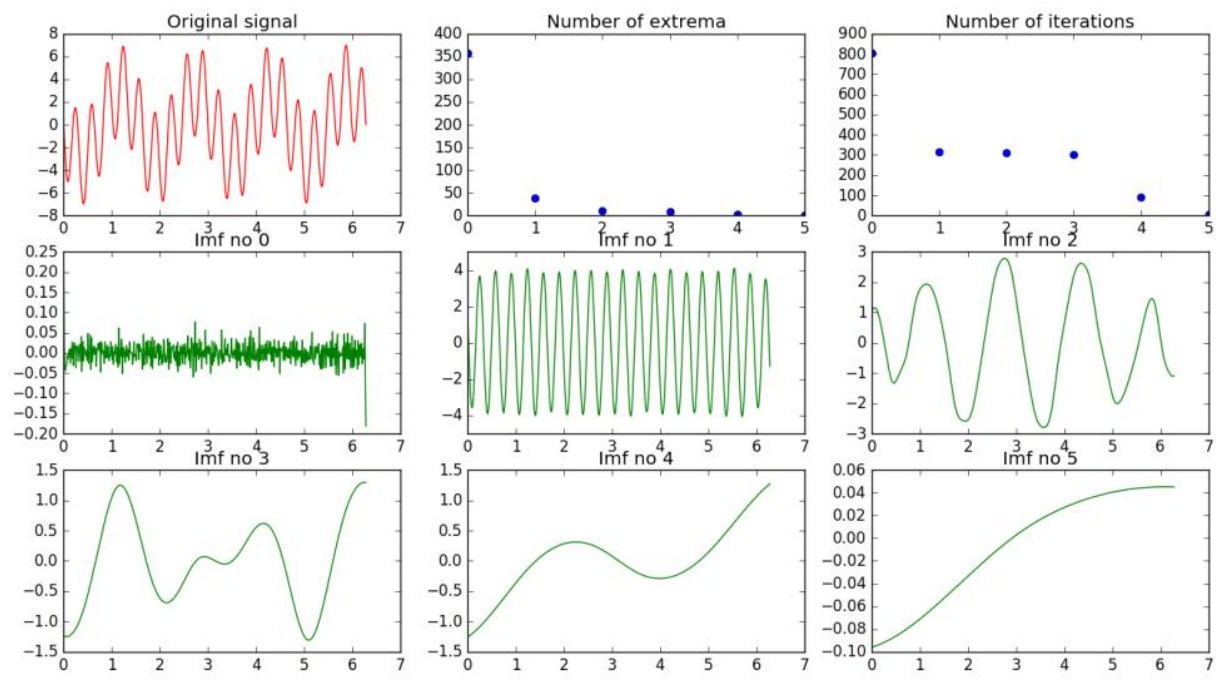

Figure-3: The original vibration signal and IMFs generated by EEMD

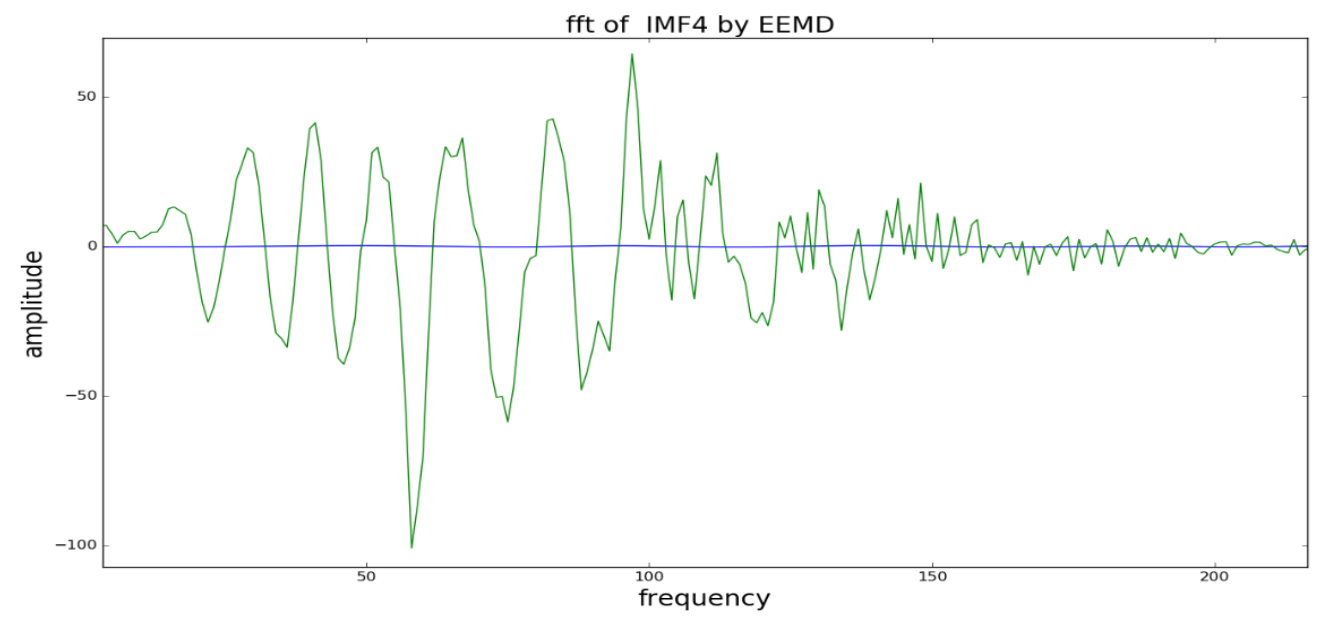

Figure-4: The fault frequency on outer race $(60 \mathrm{~Hz})$

\section{CONCLUzION}

In this paper we used EEMD technique to generate IMF for fault detection in the roller bearings. The proposed method performs in a completely data-driven manner by assessing the role played by each IMF in the diagnosis of the vibration signal collected from bearing in different operating conditions. It facilitates in detection of outer race bearing faults easily. But it has shown it's limitation, as far as roller faults are concerned. This aspect is intended be the future work.

\section{REFERENCES}

1. Design of Machines and Structures, Vol 4, No. 2 (2014) pp. 65-70. Vibration analysis Techniques for Rolling Bearing

2. Wang H, Li R, Tang G, Yuan H, Zhao Q, et al. (2014) A Compound Fault Diagnosis for Rolling Bearings Method Based on Blind Source Separation and Ensemble Empirical Mode Decomposition. PLoSONE 9(10): e109166. doi: 10.1371/journal.pone.0109166.

3. Journal of Sound and Vibration 377(2016)331-345- Convolutional Neural Network Based Fault Detection for Rotating Machinery

4. M. Grasso, S. Chatterton, P. Pennacchi, B.M. Colosimo (2016) A data-driven method to enhance vibration signal decomposition for rolling bearing fault analysis Mechanical Systems and Signal Processing, Volume 81, 15 December 2016, Pages 126-147
5. Hong, S., Zhou, Z., Zio, E. and Wang, W., An Adaptive Method for Health Trend Prediction of Rotating Bearings, Digital Signal Processing, Vol. 35, 2014, 117-123.

6. Wang, Y., Kang, S., Jiang, Y., Yang, G., Song, L., M., V.I., Classification of Fault Location and the Degree of Performance Degradation of a Rolling Bearing Based on An Improved Hypersphere-Structured Multi-Class Support Vector Machine, Mechanical Systems and Signal Processing, 29, 2012, 404-414.

\section{AUTHORSPROFILE}

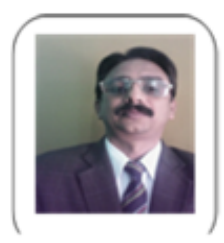

Sandip Kumar Singh is presently employed as an Associate Professor in the Department of Mechanical Engineering at V B S Purvanchal University Jaunpur (U.P.), India. He completed his B.Tech. degree from Kamla Nehru Institute of Technology (KNIT) Sultanpur (U.P.), and did M.Tech. from National Institute of Technology (N I T) Kurukshetra. He has done Ph.D. from Indian Institute of Technology (IIT BHU) Varanasi. His area of interest is Machine Learning and Structural Health Monitoring. 